\title{
FINITE ELEMENT APPROXIMATION OF THE $p$-LAPLACIAN
}

\author{
JOHN W. BARRETT AND W. B. LIU
}

\begin{abstract}
In this paper we consider the continuous piecewise linear finite element approximation of the following problem: Given $p \in(1, \infty), f$, and $g$, find $u$ such that

$$
-\nabla \cdot\left(|\nabla u|^{p-2} \nabla u\right)=f \quad \text { in } \Omega \subset \mathbb{R}^{2}, \quad u=g \text { on } \partial \Omega .
$$

The finite element approximation is defined over $\Omega^{h}$, a union of regular triangles, yielding a polygonal approximation to $\Omega$. For sufficiently regular solutions $u$, achievable for a subclass of data $f, g$, and $\Omega$, we prove optimal error bounds for this approximation in the norm $W^{1, q}\left(\Omega^{h}\right), q=p$ for $p<2$ and $q \in[1,2]$ for $p>2$, under the additional assumption that $\Omega^{h} \subseteq \Omega$. Numerical results demonstrating these bounds are also presented.
\end{abstract}

\section{INTRODUCTION}

Let $\Omega$ be a bounded open set in $\mathbb{R}^{2}$ with a Lipschitz boundary $\partial \Omega$. Given $p \in(1, \infty), f \in L^{2}(\Omega)$, and $g \in W^{1-1 / p, p}(\partial \Omega)$, we consider the following problem:

$(\mathscr{P})$ Find $u \in W_{g}^{1, p}(\Omega) \equiv\left\{v \in W^{1, p}(\Omega): v=g\right.$ on $\left.\partial \Omega\right\}$ such that

$$
\int_{\Omega}|\nabla u|^{p-2}(\nabla u, \nabla v)_{\mathbb{R}^{2}} d \Omega=\int_{\Omega} f v d \Omega \quad \forall v \in W_{0}^{1, p}(\Omega),
$$

where $|v|^{2}=(v, v)_{\mathbb{R}^{2}}$. Throughout we adopt the standard notation $W^{m, q}(D)$ for Sobolev spaces on $D$ with norm $\|\cdot\|_{W^{m, q}(D)}$ and seminorm $|\cdot|_{W^{m, q}(D)}$. We note that the seminorm $|\cdot|_{W^{1, q(D)}}$ and the norm $\|\cdot\|_{W^{1, q}(D)}$ are equivalent on $W_{0}^{1, q}(D)$.

Problem $(\mathscr{P})$ above is the weak formulation of the Dirichlet problem for the $p$-Laplacian

$$
-\nabla \cdot\left(|\nabla u|^{p-2} \nabla u\right)=f \quad \text { in } \Omega, \quad u=g \quad \text { on } \partial \Omega .
$$

The well-posedness of $(\mathscr{P})$ is well established, and one can refer to, for example, Glowinski and Marrocco [5] or the account in Ciarlet [4]. Of course, one can study more general boundary conditions and the presence of lowerorder terms in the differential operator. However, for ease of exposition, we just consider $(\mathscr{P})$, although most of our results can be adapted to more general

Received by the editor May 28, 1991 and, in revised form, March 25, 1992 and September 10, 1992.

1991 Mathematics Subject Classification. Primary 65N30.

Research supported by SERC research grant GR/F81255. 
problems. From Glowinski and Marrocco [5], or Ciarlet [4], $(\mathscr{P})$ is equivalent to the following minimization problem:

$(\mathscr{Q})$ Find $u \in W_{g}^{1, p}(\Omega)$ such that

$$
J_{\Omega}(u) \leq J_{\Omega}(v) \quad \forall v \in W_{g}^{1, p}(\Omega),
$$

where

$$
J_{\Omega}(v) \equiv \frac{1}{p} \int_{\Omega}|\nabla v|^{p} d \Omega-\int_{\Omega} f v d \Omega .
$$

It is easily established that $J_{\Omega}(\cdot)$ is strictly convex and continuous on $W_{g}^{1, p}(\Omega)$. Further, $J_{\Omega}(\cdot)$ is Gateaux differentiable with

$$
J_{\Omega}^{\prime}(u)(v) \equiv \int_{\Omega}|\nabla u|^{p-2}(\nabla u, \nabla v)_{\mathbb{R}^{2}} d \Omega-\int_{\Omega} f v d \Omega \quad \forall v \in W_{0}^{1, p}(\Omega)
$$

Hence, there exists a unique solution to $(\mathscr{Q})$, and $(\mathscr{Q})$ is equivalent to $(\mathscr{P})$, its Euler equation. In addition, we have that

$$
\|u\|_{W^{1, p}(\Omega)} \leq C\left[\|f\|_{L^{2}(\Omega)}^{1 /(p-1)}+\|g\|_{W^{1-1 / p, p}(\partial \Omega)}\right] .
$$

The problem $(\mathscr{P})$ occurs in many mathematical models of physical processes: nonlinear diffusion and filtration, see Philip [8]; power-law materials, see Atkinson and Champion [1]; and quasi-Newtonian flows, see Atkinson and Jones [2], for example.

It is the purpose of this paper to analyze the finite element approximation of $(\mathscr{P})$. Let $\Omega^{h}$ be a polygonal approximation to $\Omega$ defined by $\overline{\Omega^{h}} \equiv \bigcup_{\tau \in T^{h}} \bar{\tau}$, where $T^{h}$ is a partitioning of $\Omega^{h}$ into a finite number of disjoint open regular triangles $\tau$, each of maximum diameter bounded above by $h$. In addition, for any two distinct triangles, their closures are either disjoint, or have a common vertex, or a common side. Let $\left\{P_{j}\right\}_{j=1}^{J}$ be the vertices associated with the triangulation $T^{h}$, where $P_{j}$ has coordinates $\left(x_{j}, y_{j}\right)$. Throughout we assume that $P_{j} \in \partial \Omega^{h}$ implies $P_{j} \in \partial \Omega$, and that $\Omega^{h} \subseteq \Omega$. We note that, owing to the elliptic degeneracy of the $p$-Laplacian and the limited regularity of the solution $u$, see below, it is not a simple matter to extend the results in this paper to the case $\Omega^{h} \nsubseteq \Omega$. Associated with $T^{h}$ is the finite-dimensional space

$$
S^{h} \equiv\left\{\chi \in C\left(\overline{\Omega^{h}}\right):\left.\chi\right|_{\tau} \text { is linear } \forall \tau \in T^{h}\right\} \subset W^{1, p}\left(\Omega^{h}\right) .
$$

Let $\pi_{h}: C\left(\overline{\Omega^{h}}\right) \rightarrow S^{h}$ denote the interpolation operator such that for any $v \in$ $C\left(\overline{\Omega^{h}}\right)$, the interpolant $\pi_{h} v \in S^{h}$ satisfies $\pi_{h} v\left(P_{j}\right)=v\left(P_{j}\right), j=1, \ldots, J$. We recall the following standard approximation results. For $m=0$ or 1 , and for all $\tau \in T^{h}$, we have (a) for $q \in[1, \infty], s \in[1, \infty]$, provided $W^{2, s}(\tau) \hookrightarrow$ $W^{m, q}(\tau)$,

$$
\left|v-\pi_{h} v\right|_{W^{m, q}(\tau)} \leq C h^{2(1 / q-1 / s)} h^{2-m}|v|_{W^{2, s}(\tau)} \quad \forall v \in W^{2, s}(\tau) ;
$$

and (b) for $q>2$,

$$
\left|v-\pi_{h} v\right|_{W^{m, q}(\tau)} \leq C h^{1-m}|v|_{W^{1, q}(\tau)} \quad \forall v \in W^{1, q}(\tau) .
$$

In (1.6a) we have noted the imbedding $W^{2,1}(\tau) \hookrightarrow C(\bar{\tau})$; see, for example, p. 300 in Kufner et al. [6].

The finite element approximation of $(\mathscr{P})$ that we wish to consider is: 
$\left(\mathscr{P}^{h}\right)$ Find $u^{h} \in S_{g}^{h}$ such that

$$
\int_{\Omega^{h}}\left|\nabla u^{h}\right|^{p-2}\left(\nabla u^{h}, \nabla v^{h}\right)_{\mathbb{R}^{2}} d \Omega^{h}=\int_{\Omega^{h}} f v^{h} d \Omega^{h} \quad \forall v^{h} \in S_{0}^{h},
$$

where

$$
S_{0}^{h} \equiv\left\{\chi \in S^{h}: \chi=0 \text { on } \partial \Omega^{h}\right\}
$$

and

$$
S_{g}^{h} \equiv\left\{\chi \in S^{h}: \chi=g^{h} \text { on } \partial \Omega^{h}\right\},
$$

where $g^{h} \in S^{h}$ is chosen to approximate the Dirichlet boundary data. If $p>2$, then $u \in W^{1, p}(\Omega)$ implies $u \in C(\bar{\Omega})$, and so we set $g^{h} \equiv \pi_{h} u$. For the explicit error bounds derived in $\S 3$ for $p<2$, we assume that $u \in C(\bar{\Omega})$, and so once again set $g^{h} \equiv \pi_{h} u$. However, for the abstract analysis of this and the next section, $g^{h}$ can be arbitrary. The corresponding minimization problem is:

$\left(\mathscr{Q}^{h}\right)$ Find $u^{h} \in S_{g}^{h}$ such that

$$
J_{\Omega^{h}}\left(u^{h}\right) \leq J_{\Omega^{h}}\left(v^{h}\right) \quad \forall v^{h} \in S_{g}^{h} .
$$

The well-posedness of $\left(\mathscr{P}^{h}\right) \equiv\left(\mathscr{Q}^{h}\right)$ follows in an analogous way to that of $(\mathscr{P})$ and $(\mathscr{Q})$, see Glowinski and Marrocco [5] or Ciarlet [4], and

$$
\left\|u^{h}\right\|_{W^{1, p}\left(\Omega^{h}\right)} \leq C\left[\|f\|_{L^{2}\left(\Omega^{h}\right)}^{1 /(p-1)}+\left\|g^{h}\right\|_{W^{1, p}\left(\Omega^{h}\right)}\right] .
$$

We note that for $p=2$, problem $(\mathscr{P})$ reduces to the weak formulation of the linear Laplacian, and hence the regularity of $u$ and the finite element error analysis are well established in this case. For $p \neq 2$, the regularity of $u$ is less well established, as (1.1) is then a degenerate quasi-linear elliptic problem. It is well known, see Example 3.1 in $\S 3$, that $u$ has limited regularity for infinitely smooth data $f, g$, and $\Omega$. Therefore, there is no benefit in considering higherorder finite element approximations, and hence our restriction to continuous piecewise linears from the outset. Lieberman [7] has proved that if $\partial \Omega \in$ $C^{1, \beta}$, then $g$ is the trace of a function $\in C^{1, \gamma}(\bar{\Omega})$ for $\beta, \gamma \in(0,1)$, and if $f \in L^{\infty}(\Omega)$, then $u \in C^{1, \alpha}(\bar{\Omega})$ for some $\alpha \in(0,1)$. However, for explicit finite element error bounds one requires global regularity results on the second, or maybe higher, derivatives of $u$. Unfortunately, such results are not available at present in the literature, but it is an active area of research worldwide.

The following error bounds were proved in Glowinski and Marrocco [5] for the case $\Omega^{h} \equiv \Omega$ and $g \equiv 0$ :

If $u \in W_{0}^{1, p}(\Omega) \cap W^{2, p}(\Omega)$, then

$$
\left\|u-u^{h}\right\|_{W^{1, p}(\Omega)} \leq \begin{cases}C h^{1 /(3-p)} & \text { if } p \leq 2, \\ C h^{1 /(p-1)} & \text { if } p \geq 2,\end{cases}
$$

where throughout this paper $C$ denotes a generic positive constant independent of $h$. Chow [3], employing an approach of Tyukhtin [9], improved these error bounds. He proved that

$$
\left\|u-u^{h}\right\|_{W^{1, p}(\Omega)} \leq C\left\|u-v^{h}\right\|_{W^{1, p}(\Omega)}^{p / 2} \quad \forall v^{h} \in S_{0}^{h} \quad \text { if } p \leq 2,
$$

and

$$
\left\|u-u^{h}\right\|_{W^{1, p}(\Omega)} \leq C\left(\left\|\pi_{h} u\right\|_{W^{1, p}(\Omega)}\right)\left\|u-\pi_{h} u\right\|_{W^{1, p}(\Omega)}^{2 / p} \quad \text { if } p>2,
$$


and hence, if $u \in W_{0}^{1, p}(\Omega) \cap W^{2, p}(\Omega)$, it follows form (1.6a) that

$$
\left\|u-u^{h}\right\|_{W^{1, p}(\Omega)} \leq \begin{cases}C h^{p / 2} & \text { if } p \leq 2 \\ C h^{2 / p} & \text { if } p \geq 2\end{cases}
$$

It is the purpose of this paper to prove optimal error bounds. The layout is as follows. In the next section we prove an abstract error bound for the approximation $\left(\mathscr{P}^{h}\right)$ of $(\mathscr{P})$. In $\S 3$ we study the case $p \in(1,2)$ and prove an optimal $W^{1, p}$ error bound, that is, $O(h)$, provided that $u \in W^{3,1}(\Omega) \cap$ $C^{2,(2-p) / p}(\bar{\Omega})$. Thus, this optimal error bound requires a stronger regularity assumption on $u$ than that for the bound (1.12) in the case $p<2$. In $\S 4$ we study the case $p>2$ and first show that the bound (1.12) for $p>2$ can be achieved under the weaker regularity requirement $u \in W^{1, \infty}(\Omega) \cap W^{2,2}(\Omega)$. Second, under the additional assumption $|f| \geq \rho>0$ a.e. in $\Omega$, we prove an optimal $W^{1,4 / 3}$ error bound. We note that the above regularity requirements on $u$ for these optimal error bounds are achievable for a subclass of data $f, g$, and $\Omega$. In $\S 5$ we show that the error bounds derived in the previous sections hold for the fully practical scheme of employing numerical integration on the right-hand side of (1.7) if $f$ is sufficiently smooth. Finally, we report on some numerical examples, which confirm these optimal error bounds.

\section{AN ABSTRACT ERROR BOUND}

We first prove a lemma, which is a generalization of Lemmas 5.1, 5.2, 5.3 and 5.4 in Glowinski and Marrocco [5].

Lemma 2.1. For all $p>1$ and $\delta \geq 0$ there exist positive constants $C_{1}$ and $C_{2}$ such that for all $\xi, \eta \in \mathbb{R}^{2}, \xi \neq \eta$,

$$
\left.|| \xi\right|^{p-2} \xi-|\eta|^{p-2} \eta\left|\leq C_{1}\right| \xi-\left.\eta\right|^{1-\delta}(|\xi|+|\eta|)^{p-2+\delta}
$$

and

$$
\left(|\xi|^{p-2} \xi-|\eta|^{p-2} \eta, \xi-\eta\right)_{\mathbb{R}^{2}} \geq C_{2}|\xi-\eta|^{2+\delta}(|\xi|+|\eta|)^{p-2-\delta}
$$

Proof. The approach is similar to that in Glowinski and Marrocco [5]. For all $\xi, \eta \in \mathbb{R}^{2}, \xi \neq \eta$, let

$$
\left.G_{1}(\xi, \eta) \equiv|| \xi\right|^{p-2} \xi-|\eta|^{p-2} \eta \mid /\left[|\xi-\eta|^{1-\delta}(|\xi|+|\eta|)^{p-2+\delta}\right] .
$$

We wish to prove that $G_{1}$ is bounded above. For any $\varepsilon>0, G_{1}$ is continuous on

$$
D_{\varepsilon} \equiv\{(\xi, \eta):|\xi-\eta| \geq \varepsilon \text { and }(|\xi|+|\eta|) \leq 1 / \varepsilon\} .
$$

In addition, we note that for all $\xi, \eta \in \mathbb{R}^{2}, \xi \neq \eta$,

$$
\begin{gathered}
G_{1}(\xi, \eta) \equiv G_{1}(\eta, \xi), \quad G_{1}(\lambda \xi, \lambda \eta) \equiv G_{1}(\xi, \eta) \quad \text { for all } \lambda \in \mathbb{R}^{+}, \\
G_{1}(0, \eta)=1 \quad \text { and } \quad G_{1}(A \xi, A \eta) \equiv G_{1}(\xi, \eta) \quad \text { if } A^{T} A=I,
\end{gathered}
$$

i.e., $A$ is a rotation matrix. Therefore, without loss of generality we can take $\xi=e_{1} \equiv(1,0)$. Since

$$
G_{1}\left(e_{1}, \eta\right) \rightarrow 1 \text { as }|\eta| \rightarrow \infty,
$$

it remains to show that $\lim \sup G_{1}\left(e_{1}, \eta\right)<\infty$ as $\left|e_{1}-\eta\right| \rightarrow 0$. 
Let $\eta=(1+\rho \cos \theta, \rho \sin \theta)$. Then a simple calculation yields that

$$
\lim _{\rho \rightarrow 0} G_{1}\left(e_{1}, \eta\right)= \begin{cases}0 & \text { if } \delta>0, \\ 2^{2-p}\left\{1+p(p-2) \cos ^{2} \theta\right\}^{1 / 2} & \text { if } \delta=0 .\end{cases}
$$

Hence the desired result (2.1a).

Similarly, we prove $(2.1 \mathrm{~b})$. Let

$$
G_{2}(\xi, \eta) \equiv|\xi-\eta|^{2+\delta}(|\xi|+|\eta|)^{p-2-\delta} /\left(|\xi|^{p-2} \xi-|\eta|^{p-2} \eta, \xi-\eta\right)_{\mathbb{R}^{2}}
$$

From Glowinski and Marrocco [5] we have that

$$
\left(|\xi|^{p-2} \xi-|\eta|^{p-2} \eta, \xi-\eta\right)_{\mathbb{R}^{2}}>0 \text { if } \xi \neq \eta \text {. }
$$

Therefore, we only need to prove that $G_{2}$ is bounded above. In addition, the results (2.3) and (2.4) hold for $G_{2}$.

Setting $\eta=(1+\rho \cos \theta, \rho \sin \theta)$, a simple calculation yields that

$$
\lim _{\rho \rightarrow 0} G_{2}\left(e_{1}, \eta\right)= \begin{cases}0 & \text { if } \delta>0 \\ 2^{p-2}\left\{1+(p-2) \cos ^{2} \theta\right\}^{-1} & \text { if } \delta=0 .\end{cases}
$$

Hence the desired result (2.1b).

The inequality (2.1a) was proved in Glowinski and Marrocco [5] for $p \in$ $(1,2]$ with $\delta=2-p$, and for $p \geq 2$ with $\delta=0$; similarly, (2.1b) was proved for $p \in(1,2]$ with $\delta=0$, and for $p \geq 2$ with $\delta=p-2$.

For $p \in(1, \infty)$ and $\sigma \geq 0$ we define for any $v \in W^{1, p}\left(\Omega^{h}\right)$

$$
|v|_{(p, \sigma)} \equiv \int_{\Omega^{h}}(|\nabla u|+|\nabla v|)^{p-\sigma}|\nabla v|^{\sigma} d \Omega^{h}
$$

where $u$ is the solution of $(\mathscr{P})$. We prove the following results for later use.

Lemma 2.2. For $p \in(1, \sigma]$ we have

$$
|v|_{(p, \sigma)}^{\sigma / p} \leq|v|_{W^{1, p}\left(\Omega^{h)}\right.}^{\sigma} \leq C\left[|u|_{W^{1, p}\left(\Omega^{h}\right)}+|v|_{W^{1, p}\left(\Omega^{h}\right)}\right]^{\sigma-p}|v|_{(p, \sigma)},
$$

and for $p \in[\sigma, \infty)$,

$$
|v|_{W^{1, p}\left(\Omega^{h}\right)}^{p} \leq|v|_{(p, \sigma)} \leq C\left[|u|_{W^{1, p}\left(\Omega^{h}\right)}+|v|_{W^{1, p}\left(\Omega^{h}\right)}\right]^{p-\sigma}|v|_{W^{1, p}\left(\Omega^{h}\right)}^{\sigma} .
$$

Hence, (2.9) is well defined for $v \in W^{1, p}\left(\Omega^{h}\right)$.

Proof. Setting $w \equiv(|\nabla u|+|\nabla v|)^{p-\sigma}$, we first consider the case $p \in(1, \sigma]$. The left inequality in (2.10a) follows immediately from noting that $w \leq|\nabla v|^{p-\sigma}$. Applying Hölder's inequality, we have

$$
\begin{aligned}
|v|_{W^{1, p}\left(\Omega^{h}\right)}^{\sigma} & \equiv\left\{\int_{\Omega^{h}} w^{-p / \sigma}\left[w^{1 / \sigma}|\nabla v|\right]^{p} d \Omega^{h}\right\}^{\sigma / p} \\
& \leq\left\{\int_{\Omega^{h}} w^{-p /(\sigma-p)} d \Omega^{h}\right\}^{(\sigma-p) / p}|v|_{(p, \sigma)} \\
& =\left\{\int_{\Omega^{h}}(|\nabla u|+|\nabla v|)^{p} d \Omega^{h}\right\}^{(\sigma-p) / p}|v|_{(p, \sigma)} .
\end{aligned}
$$

The right inequality in (2.10a) follows by noting that for all $\gamma \in[0, \infty)$ there exists $C_{\gamma}>0$ such that $|a+b|^{\gamma} \leq C_{\gamma}\left(|a|^{\gamma}+|b|^{\gamma}\right)$ for all $a, b \in \mathbb{R}$.

The inequalities $(2.10 \mathrm{~b})$ can be proved in a similar manner.

The next theorem is the natural generalization of the result in $\S 7$ of Chow [3]. We use the minimization property of $u^{h}$ and Lemma 2.1, whereas Chow uses the Glowinski and Marrocco version of Lemma 2.1. 
Theorem 2.1. Let $u$ and $u^{h}$ be the unique solutions of $(\mathscr{P}) \equiv(\mathscr{Q})$ and $(\mathscr{P} h) \equiv$ $\left(\mathscr{Q}^{h}\right)$, respectively. Then for any $\delta_{1} \in[0,2)$ and $\delta_{2} \geq 0$, and any $v^{h} \in S_{g}^{h}$, it follows that

$$
\left|u-u^{h}\right|_{\left(p, 2+\delta_{2}\right)} \leq C\left|u-v^{h}\right|_{\left(p, 2-\delta_{1}\right)} .
$$

Proof. We have for any $v^{h} \in S_{g}^{h}$ that

$$
\begin{aligned}
J_{\Omega^{h}}\left(v^{h}\right)-J_{\Omega^{h}}(u)= & \int_{0}^{1} J_{\Omega^{h}}^{\prime}\left(u+s\left(v^{h}-u\right)\right)\left(v^{h}-u\right) d s \\
= & \int_{0}^{1}\left[J_{\Omega^{h}}^{\prime}\left(u+s\left(v^{h}-u\right)\right)\left(\left[u+s\left(v^{h}-u\right)\right]-u\right)\right. \\
& \left.\quad-J_{\Omega^{h}}^{\prime}(u)\left(\left[u+s\left(v^{h}-u\right)\right]-u\right)\right] \frac{d s}{s} \\
& +J_{\Omega^{h}}^{\prime}(u)\left(v^{h}-u\right) \\
\equiv & A\left(v^{h}\right)+J_{\Omega^{h}}^{\prime}(u)\left(v^{h}-u\right),
\end{aligned}
$$

where from (1.3)

$$
\begin{aligned}
A\left(v^{h}\right) \equiv \int_{0}^{1}\left[\int _ { \Omega ^ { h } } \left\{\left[\left|\nabla\left(u+s\left(v^{h}-u\right)\right)\right|^{p-2}\right.\right.\right. \\
\left.\left.\left.\times \nabla\left(u+s\left(v^{h}-u\right)\right)-|\nabla u|^{p-2} \nabla u\right] \nabla\left(v^{h}-u\right)\right\} d \Omega^{h}\right] d s .
\end{aligned}
$$

From $(2.12 b)$ and $(2.1 a)$ we have that

$$
\begin{array}{rlrl}
\left|A\left(v^{h}\right)\right| & \leq C_{1} \int_{0}^{1} s^{1-\delta_{1}} \int_{\Omega^{h}}\left(\left|\nabla\left[u+s\left(v^{h}-u\right)\right]\right|+|\nabla u|\right)^{p-2+\delta_{1}} \\
& \leq C\left|u-v^{h}\right|_{\left(p, 2-\delta_{1}\right)}, & \times\left|\nabla\left(v^{h}-u\right)\right|^{2-\delta_{1}} d \Omega^{h} d s
\end{array}
$$

where we have noted that for all $v_{1}, v_{2}$, and $s \in[0,1]$

$$
\frac{1}{2} s\left(\left|\nabla v_{1}\right|+\left|\nabla v_{2}\right|\right) \leq\left|\nabla\left[v_{1}+s v_{2}\right]\right|+\left|\nabla v_{1}\right| \leq 2\left(\left|\nabla v_{1}\right|+\left|\nabla v_{2}\right|\right) \text {. }
$$

From $(2.12 b),(2.1 b)$, and $(2.14)$ we have that

$$
\begin{aligned}
\left|A\left(v^{h}\right)\right| & \geq C_{2} \int_{0}^{1} s^{1+\delta_{2}} \int_{\Omega^{h}}\left(\left|\nabla\left[u+s\left(v^{h}-u\right)\right]\right|+|\nabla u|\right)^{p-2+\delta_{2}} \\
& \times C\left|u-v^{h}\right|_{\left(p, 2+\delta_{2}\right)} .
\end{aligned}
$$

From (1.8) and (2.12) we have that for all $v^{h} \in S_{g}^{h}$

$$
\begin{aligned}
A\left(u^{h}\right)+J_{\Omega^{h}}^{\prime}(u)\left(u^{h}-u\right) & \equiv J_{\Omega^{h}}\left(u^{h}\right)-J_{\Omega^{h}}(u) \leq J_{\Omega^{h}}\left(v^{h}\right)-J_{\Omega^{h}}(u) \\
& \equiv A\left(v^{h}\right)+J_{\Omega^{h}}^{\prime}(u)\left(v^{h}-u\right) .
\end{aligned}
$$

Therefore, it follows from (2.16), (2.13), and (2.15) that

$$
\left|u-u^{h}\right|_{\left(p, 2+\delta_{2}\right)} \leq C\left|u-v^{h}\right|_{\left(p, 2-\delta_{1}\right)}+J_{\Omega^{h}}^{\prime}(u)\left(v^{h}-u^{h}\right) .
$$


As $\Omega^{h}$ is Lipschitz, $\Omega^{h} \subseteq \Omega$ and $\chi \equiv v^{h}-u^{h} \in S_{0}^{h}$, we can extend $\chi$ to be zero on $\Omega \backslash \Omega^{h}$. Denoting this extension by $\hat{\chi}$, we have that $\hat{\chi} \in W_{0}^{1, p}(\Omega)$ and hence from $(\mathscr{P})$ that $J_{\Omega^{h}}^{\prime}(u)(\chi) \equiv J_{\Omega}^{\prime}(u)(\hat{\chi})=0$. Therefore, the desired result (2.11) follows from (2.17).

\section{ERROR BOUNDS FOR $p \in(1,2)$}

Assuming that $u \in W^{2,1}(\Omega)$, which implies that $u \in C(\bar{\Omega})$, we can set $g^{h} \equiv \pi_{h} u$ in (1.7c). Choosing $\delta_{2}=0$ in (2.11) and noting (2.10a), (1.4), (1.9), and $(1.6 \mathrm{a})$, we have for all $\delta_{1} \in[0,2)$ and for all $v^{h} \in S_{g}^{h}$

$$
\left|u-u^{h}\right|_{W^{1, p}\left(\Omega^{h}\right)}^{2} \leq C\left|u-u^{h}\right|_{(p, 2)} \leq C\left|u-v^{h}\right|_{\left(p, 2-\delta_{1}\right)} \text {. }
$$

Choosing $\delta_{1}=2-p$ and noting (2.10a) yield that for all $v^{h} \in S_{g}^{h}$

$$
\left|u-u^{h}\right|_{W^{1, p}\left(\Omega^{h}\right)}^{2} \leq C\left|u-v^{h}\right|_{(p, p)} \leq C\left|u-v^{h}\right|_{W^{1, p}\left(\Omega^{h}\right)}^{p} .
$$

From a Poincaré inequality we have for all $q \in[1, \infty)$, for all $v \in W^{1, q}(\Omega)$, and for all $v^{h}, w^{h} \in S_{g}^{h}$ that

$$
\left\|v-w^{h}\right\|_{W^{1, q}\left(\Omega^{h}\right)} \leq C\left\|v-v^{h}\right\|_{W^{1, q}\left(\Omega^{h}\right)}+C\left|v-w^{h}\right|_{W^{1, q}\left(\Omega^{h}\right)} .
$$

Hence, from (3.2) with $v^{h} \equiv \pi_{h} u,(3.3)$, and (1.6a) we have that

$$
\begin{aligned}
\left\|u-u^{h}\right\|_{W^{1, p}\left(\Omega^{h}\right)} & \leq C\left\|u-\pi_{h} u\right\|_{W^{1, p}\left(\Omega^{h}\right)}+C\left|u-\pi_{h} u\right|_{W^{1, p}\left(\Omega^{h}\right)}^{p / 2} \\
& \leq C h^{p / 2} \text { if } u \in W^{2, p}(\Omega),
\end{aligned}
$$

the generalization of the results (1.11a) and (1.12), for $p<2$, of Chow [3] to the case of nonhomogeneous boundary data $g$ and $\Omega^{h} \subseteq \Omega$. Below we prove an optimal $W^{1, p}$ error bound for sufficiently regular $u$, based on choosing $\delta_{1}=0$ in (3.1).

Lemma 3.1. Let $\alpha \in(-1,0)$. If $v \in W^{2,1}(\Omega)$, then

$$
\int_{\Omega}|v|^{\alpha}|\nabla v|^{2} d \Omega<\infty
$$

Proof. We have that

$$
\begin{aligned}
& \int_{\Omega}|v|^{\alpha}\left|v_{x}\right|^{2} d x d y \equiv \frac{1}{\alpha+1} \int_{\Omega}\left(\operatorname{sign}(v)|v|^{\alpha+1}\right)_{x} v_{x} d x d y \\
& \quad \equiv \frac{1}{\alpha+1}\left\{\int_{\partial \Omega} \operatorname{sign}(v)|v|^{\alpha+1} v_{x} d y-\int_{\Omega} \operatorname{sign}(v)|v|^{\alpha+1} v_{x x} d x d y\right\}
\end{aligned}
$$

and a similar identity with $v_{x}$ replaced by $v_{y}$. The desired result (3.5) then follows from the imbedding $W^{2,1}(\Omega) \hookrightarrow C(\bar{\Omega})$ and the trace inequality $\|\cdot\|_{L^{1}(\partial \Omega)} \leq C\|\cdot\|_{W^{1,1}(\Omega)}$.

Theorem 3.1. If $u \in W^{3,1}(\Omega) \cap C^{2, \alpha}(\bar{\Omega})$, with $\alpha>0$, then it follows that

$$
\left\|u-u^{h}\right\|_{W^{1, p}\left(\Omega^{h}\right)}^{2} \leq C\left[h^{2}+h^{p(1+\alpha)}\right],
$$

and hence, if $u \in W^{3,1}(\Omega) \cap C^{2,(2-p) / p}(\bar{\Omega})$, then

$$
\left\|u-u^{h}\right\|_{W^{1, p}\left(\Omega^{h}\right)} \leq C h .
$$


Proof. As $u \in C^{2, \alpha}(\bar{\Omega})$, we have from (1.6a) that for all $\tau \in T^{h}$ and for all $(x, y) \in \bar{\tau}$

$$
\left|\nabla\left(u-\pi_{h} u\right)(x, y)\right| \leq C h|H[u]|_{L^{\infty}(\tau)} \leq C h H[u](x, y)+C h^{1+\alpha},
$$

where $H[u] \equiv\left|u_{x x}\right|+\left|u_{x y}\right|+\left|u_{y y}\right|$.

It is easy to check that the function $q(t) \equiv(a+t)^{p-2} t^{2}$ with $a \geq 0$ is increasing on $\mathbb{R}^{+}$and hence that $q\left(\left|t_{1}+t_{2}\right|\right) \leq 2\left[q\left(\left|t_{1}\right|\right)+q\left(\left|t_{2}\right|\right)\right]$ for all $t_{1}, t_{2} \in$ $\mathbb{R}$. Therefore, we have from (3.1) with $\delta_{1}=0$ and $v^{h} \equiv \pi_{h} u,(3.7)$, and the above that

$$
\begin{aligned}
\left|u-u^{h}\right|_{W^{1, p}\left(\Omega^{h}\right)}^{2} \leq & C \int_{\Omega^{h}}\left(|\nabla u|+\left|\nabla\left(u-\pi_{h} u\right)\right|\right)^{p-2}\left|\nabla\left(u-\pi_{h} u\right)\right|^{2} d \Omega^{h} \\
\leq & C h^{2} \int_{\Omega^{h}}(|\nabla u|+C h H[u])^{p-2}(H[u])^{2} d \Omega^{h} \\
& +C h^{2(1+\alpha)} \int_{\Omega^{h}}\left(|\nabla u|+C h^{1+\alpha}\right)^{p-2} d \Omega^{h} \\
\leq & C h^{p(1+\alpha)}+C h^{2} \int_{\Omega^{h}}(|\nabla u|)^{p-2}(H[u])^{2} d \Omega^{h} .
\end{aligned}
$$
that

Setting $v_{1} \equiv u_{x}$ and $v_{2} \equiv u_{y}$, we have from (3.5), as $v_{1}, v_{2} \in W^{2,1}(\Omega)$,

$$
\text { (3.9) } \begin{gathered}
\int_{\Omega^{h}}(|\nabla u|)^{p-2}(H[u])^{2} d \Omega^{h} \leq C \int_{\Omega^{h}}\left(v_{1}^{2}+v_{2}^{2}\right)^{(p-2) / 2}\left(\left|\nabla v_{1}\right|^{2}+\left|\nabla v_{2}\right|^{2}\right) d \Omega^{h} \\
\leq C \int_{\Omega^{h}}\left[\left|v_{1}\right|^{p-2}\left|\nabla v_{1}\right|^{2}+\left|v_{2}\right|^{p-2}\left|\nabla v_{2}\right|^{2}\right] d \Omega^{h}<\infty .
\end{gathered}
$$

Combining (3.8) and (3.9) yields the result (3.6a) and hence (3.6b) with $\|\cdot\|_{W^{1, p}\left(\Omega^{h}\right)}$ replaced by $|\cdot|_{W^{1, p}\left(\Omega^{h}\right)}$. The results (3.6) then follow by noting (3.3), (1.6a), and that $u \in W^{3,1}(\Omega)$ implies $u \in W^{2, p}(\Omega)$.

We note that one can prove (3.6b) under alternative regularity requirements on $u$, e.g., $u \in W^{3, p}(\Omega)$. However, we will not exploit this here. We now show that the regularity requirements on $u$ in Theorem 3.1 hold for a model problem.

Example 3.1. We consider a radially symmetric version of problem $(\mathscr{P})$. Let $\Omega \equiv\{r: r<1\}, f(x, y) \equiv F(r), f \in L^{q}(\Omega)$ for $q>2$, and $g$ be constant, where $r \equiv\left(x^{2}+y^{2}\right)^{1 / 2}$. Then

$$
\begin{gathered}
u(x, y) \equiv U(r) \equiv-\int_{r}^{1} \operatorname{sign}(Z(t))|Z(t)|^{1 /(p-1)} d t+g, \\
U^{\prime \prime} \equiv\left(|Z|^{(2-p) /(p-1)} Z^{\prime}\right) /(p-1)
\end{gathered}
$$

and

$$
U^{\prime \prime \prime} \equiv C_{1} \operatorname{sign}(Z)|Z|^{(3-2 p) /(p-1)}\left(Z^{\prime}\right)^{2}+C_{2}|Z|^{(2-p) /(p-1)} Z^{\prime \prime}
$$

for some constants $C_{i}$, where

$$
z(x, y) \equiv Z(r) \equiv\left(\left|U^{\prime}\right|^{p-2} U^{\prime}\right)(r)=-\frac{1}{r} \int_{0}^{r} t F(t) d t .
$$


It is a simple matter to deduce from $(3.10 \mathrm{~d})$ that

$$
f \in C^{0, q}(\bar{\Omega}) \Rightarrow z \in C^{1, q}(\bar{\Omega}) \quad \forall q \in[0,1]
$$

and

$$
f \in W^{1, q}(\Omega) \text { for } q>1 \Rightarrow z \in W^{2,1}(\Omega) \Rightarrow z \in C(\bar{\Omega}) .
$$

It follows from (3.10b) and (3.11a) that

$$
\begin{aligned}
& p \in\left(1, \frac{3}{2}\right] \text { and } f \in C^{0, \beta}(\bar{\Omega}) \text { for } \beta \in[0,1] \Rightarrow u \in C^{2, \beta}(\bar{\Omega}), \\
& p \in\left[\frac{3}{2}, 2\right) \text { and } f \in C^{0, \beta}(\bar{\Omega}) \text { for } \beta \in[0,(2-p) /(p-1)] \\
& \Rightarrow u \in C^{2, \beta}(\bar{\Omega}),
\end{aligned}
$$

and from $(3.10 \mathrm{c}),(3.11 \mathrm{~b})$, and Lemma 3.1 that

$$
p \in(1,2) \text { and } f \in W^{1, q}(\Omega) \text { for } q>1 \Rightarrow u \in W^{3,1}(\Omega) .
$$

\section{ERROR BOUNDS FOR $p>2$}

Let $g^{h} \equiv \pi_{h} u$ in (1.7c). From (2.11) with $\delta_{1}=0$ and $\delta_{2}=p-2,(2.10 \mathrm{~b})$, (1.4), (1.9), and (1.6b) it follows that

(4.1) $\left|u-u^{h}\right|_{W^{1, p}\left(\Omega^{h}\right)}^{p} \leq\left|u-u^{h}\right|_{(p, p)} \leq C\left|u-\pi_{h} u\right|_{(p, 2)} \leq C\left|u-\pi_{h} u\right|_{W^{1, p}\left(\Omega^{h}\right)}^{2}$, and hence it follows from (3.3) and (1.6a) that

$$
\begin{aligned}
\left\|u-u^{h}\right\|_{W^{1, p}\left(\Omega^{h}\right)} & \leq C\left\|u-\pi_{h} u\right\|_{W^{1, p}\left(\Omega^{h}\right)}+C\left|u-\pi_{h} u\right|_{W^{1, p}\left(\Omega^{h}\right)}^{2 / p} \\
& \leq C h^{2 / p} \quad \text { if } u \in W^{2, p}(\Omega),
\end{aligned}
$$

the generalization of the results (1.11b) and (1.12), for $p>2$, of Chow [3] to the case of nonhomogeneous boundary data $g$ and $\Omega^{h} \subseteq \Omega$. Alternatively, assuming $u \in W^{1, \infty}(\Omega)$, we have from (2.11) with $\delta_{1}=2-s, s \in[1,2]$, and $\delta_{2}=p-2$ and (1.6b) that

$$
\left|u-u^{h}\right|_{W^{1, p}\left(\Omega^{h}\right)}^{p} \leq\left|u-u^{h}\right|_{(p, p)} \leq C\left|u-\pi_{h} u\right|_{(p, s)} \leq C\left|u-\pi_{h} u\right|_{W^{1, s}\left(\Omega^{h}\right)}^{s}
$$

In addition, we note from (1.6b) that for $u \in W^{1, \infty}(\Omega)$

$$
\left\|u-\pi_{h} u\right\|_{W^{1, q}\left(\Omega^{h}\right)} \leq C\left\|u-\pi_{h} u\right\|_{W^{1, s}\left(\Omega^{h}\right)}^{s / q} \text { if } q>s .
$$

Hence, it follows from (4.3), (3.3), (4.4), and (1.6a) that if $u \in W^{1, \infty}(\Omega) \cap$ $W^{2, s}(\Omega), s \in[1,2]$, then

$$
\begin{aligned}
\left\|u-u^{h}\right\|_{W^{1, p}\left(\Omega^{h}\right)} & \leq C\left\|u-\pi_{h} u\right\|_{W^{1, p}\left(\Omega^{h}\right)}+C\left|u-\pi_{h} u\right|_{W^{1, s}\left(\Omega^{h}\right)}^{s / p} \\
& \leq C\left\|u-\pi_{h} u\right\|_{W^{1, s}\left(\Omega^{h}\right)}^{s / p} \leq C h^{s / p} .
\end{aligned}
$$

Choosing $f \equiv 1$ and $g \equiv 0$ in Example 3.1 yields that $u(x, y) \equiv$ $C\left(1-r^{p /(p-1)}\right)$, and so $u \in W^{2, s}(\Omega)$ only if $s<2(p-1) /(p-2)$. Therefore, in general $u$ rarely belongs to $W^{2, p}(\Omega)$ in order for $(4.2 \mathrm{~b})$ to guarantee that the error converges at least at the rate of $h^{2 / p}$ in $W^{1, p}$. However, from (4.5b) we see that this rate is ensured under the far weaker regularity requirement of $u \in W^{1, \infty}(\Omega) \cap W^{2,2}(\Omega)$, and this is satisfied by the example above.

Below we prove error bounds in weaker norms, $\|\cdot\|_{W^{1, q}\left(\Omega^{h}\right)}$ with $q \in[1, p)$. 
Lemma 4.1. For all $t \in[2, p]$ and $q \in[1, t]$ for which

$$
\int_{\Omega}|\nabla u|^{-(p-t) q /(t-q)} d \Omega<\infty \quad \text { if } q \in[1, t)
$$

and

$$
|\nabla u|^{-(p-t)} \in L^{\infty}(\Omega) \quad \text { if } q=t,
$$

we have for $u \in W^{1, \infty}(\Omega) \cap W^{2, s}(\Omega)$ with $s \in[1,2]$ that

$$
\left\|u-u^{h}\right\|_{W^{1, q}\left(\Omega^{h}\right)} \leq C h^{s / t} .
$$

Proof. Choosing $\delta_{1}=2-s$ and $\delta_{2}=t-2$ in (2.11), noting (4.3) and (4.6), and applying a Hölder inequality, we obtain that

$$
\left|u-u^{h}\right|_{W^{1, q\left(\Omega^{h}\right)}}^{t} \leq C\left|u-u^{h}\right|_{(p, t)} \leq C\left|u-\pi_{h} u\right|_{(p, s)} \leq C\left|u-\pi_{h} u\right|_{W^{1, s}\left(\Omega^{h}\right)}^{s} .
$$

The desired result (4.7) then follows from (4.8), (1.6a), (3.3), and (4.4).

To improve on the $h^{s / p}$ convergence rate for the error in (4.5b), we wish to take $t \in[2, p)$, which gives rise to the restrictions (4.6) on $u$; that is, we require $\{(x, y) \in \Omega:|\nabla u(x, y)|=0\}$ to have zero measure and a growth condition on $|\nabla u|^{-1}$. From inspection we see that the weakest growth restriction on $|\nabla u|^{-1}$ for a fixed $t$ is needed when $q=1$. We now look for sufficient conditions on $u$ and the data $f$ in order for these restrictions to hold.

Lemma 4.2. If $u \in W^{1, \infty}(\Omega) \cap W^{2, s}(\Omega), s \in[1, \infty]$, then there exists an $M \in L^{s}(\Omega)$ such that

$$
|f| \leq M|\nabla u|^{p-2} \text { a.e. in } \Omega \text {. }
$$

Proof. Let $\nabla u \equiv\left(v_{1}, v_{2}\right) \in\left[W^{1, s}(\Omega)\right]^{2}$ and $v \equiv\left(v_{1}^{2}+v_{2}^{2}\right)^{1 / 2} \equiv|\nabla u| \in L^{\infty}(\Omega)$. As $\left|v_{1} / v\right|+\left|v_{2} / v\right|$ is bounded and $\nabla v \equiv\left(v_{1} \nabla v_{1}+v_{2} \nabla v_{2}\right) / v$, it follows that $v \in W^{1, s}(\Omega)$. In addition, we have that

$$
\begin{aligned}
f & =-\operatorname{div}\left(v^{p-2} v_{1}, v^{p-2} v_{2}\right) \\
& =-v^{p-2}\left\{\left[\left(v_{1}\right)_{x}+\left(v_{2}\right)_{y}\right]+(p-2)\left[v_{1} v_{x}+v_{2} v_{y}\right] / v\right\} .
\end{aligned}
$$

Hence the desired result (4.9).

Under the assumption that $\{(x, y) \in \Omega: f(x, y)=0\}$ has zero measure, the inequality (4.9), for example, yields for $t \geq 2$ and $1 \leq q<t<p$ that

$$
\int_{\Omega}|\nabla u|^{-(p-t) q /(t-q)} d \Omega \leq \int_{\Omega}[M /|f|]^{(p-t) q /[(p-2)(t-q)]} d \Omega .
$$

Therefore, with $M \in L^{s}(\Omega)$, for a given $s \in[1, \infty]$, and imposing a growth condition on $|f|^{-1}$, one can choose appropriate $t$ and $q$ so that (4.6a) and hence (4.7) hold. Below we give an example of such a result.

Theorem 4.1. Let $u \in W^{1, \infty}(\Omega) \cap W^{2, s}(\Omega), s \in[1, \infty]$. If $|f|^{-\gamma} \in L^{1}(\Omega)$ for some $\gamma \in(0, \infty)$, or if $|f|^{-1} \in L^{\infty}(\Omega)$ we set $\gamma=\infty$, then we have for $q \in[1, p)$ that

$$
\left\|u-u^{h}\right\|_{W^{1, q}\left(\Omega^{h}\right)} \leq \begin{cases}C h^{2 / t} & \text { if } s \geq 2 \\ C h^{s / t} & \text { if } s \in[1,2)\end{cases}
$$


where

$$
t=\max \{2, q[(s+\gamma) p+(p-2) \gamma s] /[(s+\gamma) q+(p-2) \gamma s]\} .
$$

Proof. First a simple calculation yields that $t$ satisfying $(4.12 \mathrm{~b})$ is such that $t \in[2, p)$ and $t>q$. Setting

$$
\eta \equiv q(p-t) /[(p-2)(t-q)],
$$

we conclude that $\eta \leq \gamma s /(s+\gamma)$ and hence $s \eta \leq \gamma(s-\eta)$, and if $\gamma$ is finite then $\eta<s$. Therefore, from (4.11), the assumptions on $f$ and Hölder's inequality we have

$$
\begin{aligned}
\int_{\Omega}|\nabla u|^{-(p-t) q /(t-q)} d \Omega & \leq \int_{\Omega}\left(M|f|^{-1}\right)^{\eta} d \Omega \\
\leq & \left(\int_{\Omega} M^{s} d \Omega\right)^{\eta / s}\left(\int_{\Omega}|f|^{-\eta s /(s-\eta)} d \Omega\right)^{(s-\eta) / s} \leq C .
\end{aligned}
$$

Similarly, (4.13) holds if $\gamma$ is infinite, as $\eta \leq s$. The desired result (4.12a) then follows from (4.6a) and (4.7).

We note that for fixed $q, \gamma$, and $s$ the right-hand side of $(4.12 \mathrm{~b})$ tends to $\max \{(2, q[(s+\gamma)+\gamma s] / \gamma s\}$ as $p \rightarrow \infty$. Therefore, the error bound (4.12a) does not degenerate as $p \rightarrow \infty$, unlike (4.2b) and (4.5b).

Corollary 4.1. Let $u \in W^{1, \infty}(\Omega) \cap W^{2, s}(\Omega), s \in[1, \infty]$. Suppose that there exists a constant $\rho>0$ such that $|f| \geq \rho$ a.e. in $\Omega$; then for $q \in[1, p)$ we have that

$$
\left\|u-u^{h}\right\|_{W^{1, q}\left(\Omega^{h}\right)} \leq \begin{cases}C h^{2 / t} & \text { if } s \geq 2, \\ C h^{s / t} & \text { if } s \in[1,2)\end{cases}
$$

where

$$
t=\max \{2, q[p+(p-2) s] /[q+(p-2) s]\} .
$$

Hence, we have that for $q=2 s /(1+s)$

$$
\left\|u-u^{h}\right\|_{W^{1, q}\left(\Omega^{h}\right)} \leq \begin{cases}C h & \text { if } s \geq 2, \\ C h^{s / 2} & \text { if } s \in[1,2) .\end{cases}
$$

Proof. The result $(4.14 \mathrm{a}, \mathrm{b})$ follows directly from setting $\gamma=\infty$ in (4.12). The result $(4.14 \mathrm{c})$ then follows from $(4.14 \mathrm{a}, \mathrm{b})$ by noting that $t=2$ if $q=$ $2 s /(1+s)$.

\section{NUMERICAL EXAMPLES}

The standard Galerkin method analyzed in the previous sections requires the term $\int_{\Omega^{h}} f v^{h} d \Omega^{h}$ for all $v^{h} \in S_{0}^{h}$ to be integrated exactly. This is difficult in practice, and it is computationally more convenient to consider a scheme where numerical integration is applied to this term. With $\overline{\Omega^{h}} \equiv \bigcup_{\tau \in T^{h}} \bar{\tau}$ and $\left\{a_{i}\right\}_{i=1}^{3}$ being the vertices of a triangle $\tau$, we define the quadrature rule

$$
Q_{\tau}(v) \equiv \frac{1}{3} \operatorname{meas}(\tau) \sum_{i=1}^{3} v\left(a_{i}\right) \equiv \int_{\tau} \pi_{h} v d \tau
$$


approximating $\int_{\tau} v d \tau$ for $v \in C(\bar{\tau})$. Then, for $v, w \in C\left(\overline{\Omega^{h}}\right)$, we set

$$
(v, w)^{h} \equiv \sum_{\tau \in T^{h}} Q_{\tau}(v w) \equiv \int_{\Omega^{h}} \pi_{h}(v w) d \Omega^{h}
$$

as an approximation to $\int_{\Omega^{h}} v w d \Omega^{h}$.

The fully practical finite element approximation of $(\mathscr{P})$ that we wish to consider is:

$\left(\widehat{\mathscr{P}}^{h}\right)$ Find $\hat{u}^{h} \in S_{g}^{h}$ such that

$$
\int_{\Omega^{h}}\left|\nabla \hat{u}^{h}\right|^{p-2}\left(\nabla \hat{u}^{h}, \nabla v^{h}\right)_{\mathbb{R}^{2}} d \Omega^{h}=\left(f, v^{h}\right)^{h} \quad \forall v^{h} \in S_{0}^{h} .
$$

The corresponding minimization problem is:

$\left(\widehat{Q}^{h}\right)$ Find $\hat{u}^{h} \in S_{g}^{h}$ such that

$$
\widehat{J}_{\Omega^{h}}\left(\hat{u}^{h}\right) \leq \widehat{J}_{\Omega^{h}}\left(v^{h}\right) \quad \forall v^{h} \in S_{g}^{h},
$$

where

$$
\widehat{J}_{\Omega^{h}}\left(v^{h}\right) \equiv \frac{1}{p} \int_{\Omega^{h}}\left|\nabla v^{h}\right|^{p} d \Omega^{h}-\left(f, v^{h}\right)^{h} .
$$

The well-posedness of $\left(\widehat{\mathscr{P}}^{h}\right) \equiv\left(\widehat{\mathscr{Q}}^{h}\right)$ follows in an analogous way to that of $(\mathscr{P})$ and $(\mathscr{Q})$, and

$$
\left\|\hat{u}^{h}\right\|_{W^{1, p}\left(\Omega^{h}\right)} \leq C\left[\|f\|_{L^{\infty}\left(\Omega^{h}\right)}^{1 /(p-1)}+\left\|g^{h}\right\|_{W^{1, p}\left(\Omega^{h}\right)}\right] .
$$

We now bound the error $\hat{u}^{h}-u$. First we have the analogue of Theorem 2.1. Theorem 5.1. Let $u$ and $\hat{u}^{h}$ be the unique solutions of $(\mathscr{P}) \equiv(\mathscr{Q})$ and $\left(\hat{\mathscr{P}}^{h}\right) \equiv$ $\left(\widehat{Q}^{h}\right)$, respectively. Let $f \in W^{1, \infty}(\Omega) \cap W^{2,2}(\Omega)$. Then for any $\delta_{1} \in[0,2)$ and $\delta_{2} \geq 0$, and any $v^{h} \in S_{g}^{h}$, it follows that

$$
\left|u-\hat{u}^{h}\right|_{\left(p, 2+\delta_{2}\right)} \leq C\left|u-v^{h}\right|_{\left(p, 2-\delta_{1}\right)}+C h^{2}\left\|v^{h}-\hat{u}^{h}\right\|_{W^{1,1}\left(\Omega^{h}\right)} .
$$

Proof. The proof follows exactly that of Theorem 2.1 with $\hat{u}^{h}$ and $\widehat{J}_{\Omega^{h}}$ instead of $u^{h}$ and $J_{\Omega^{h}}$ in (2.16). However, whereas $J_{\Omega^{h}}^{\prime}(u)\left(v^{h}-u^{h}\right)=0$, we now have for all $v^{h} \in S_{g}^{h}$

$$
\begin{aligned}
\widehat{J}_{\Omega^{h}}^{\prime}(u)\left(v^{h}-\hat{u}^{h}\right) \equiv & J_{\Omega^{h}}^{\prime}(u)\left(v^{h}-\hat{u}^{h}\right) \\
& -\int_{\Omega^{h}} f\left(v^{h}-\hat{u}^{h}\right) d \Omega^{h}+\left(f, v^{h}-\hat{u}^{h}\right)^{h} \\
\equiv & -\int_{\Omega^{h}} f\left(v^{h}-\hat{u}^{h}\right) d \Omega^{h}+\left(f, v^{h}-\hat{u}^{h}\right)^{h}
\end{aligned}
$$

and

$$
\begin{aligned}
& \left|\int_{\Omega^{h}} f\left(v^{h}-\hat{u}^{h}\right) d \Omega^{h}-\left(f, v^{h}-\hat{u}^{h}\right)^{h}\right| \leq C\left\|\left(I-\pi_{h}\right)\left[f\left(v^{h}-\hat{u}^{h}\right)\right]\right\|_{L^{1}\left(\Omega^{h}\right)} \\
& \quad \leq C h^{2}\left|f\left(v^{h}-\hat{u}^{h}\right)\right|_{W^{2,1}\left(\Omega^{h}\right)} \leq C h^{2}\left\|v^{h}-\hat{u}^{h}\right\|_{W^{1,1}\left(\Omega^{h}\right)}
\end{aligned}
$$

provided $f \in W^{1, \infty}(\Omega) \cap W^{2,2}(\Omega)$. Hence, we obtain the desired result (5.6). 
In particular, assuming $u \in W^{2,1}(\Omega)$ if $p<2$, we have for $v^{h} \equiv g^{h} \equiv \pi_{h} u$ that for any $\delta_{1} \in[0,2)$ and $\delta_{2} \geq 0$

$$
\left|u-\hat{u}^{h}\right|_{\left(p, 2+\delta_{2}\right)} \leq C\left|u-\pi_{h} u\right|_{\left(p, 2-\delta_{1}\right)}+C h^{2} .
$$

Hence, it is a simple matter to check that the results of the previous sections hold for $\hat{u}^{h}$ as well as $u^{h}$ if $f \in W^{1, \infty}(\Omega) \cap W^{2,2}(\Omega)$. We note that this constraint on $f$ can be weakened and is imposed here for ease of exposition only.

We now report on some numerical results with the fully practical approximation (5.3). For computational ease we took $\Omega$ to be the square $[0,1] \times[0,1]$. This was partitioned into uniform right-angled triangles by dividing it first into equal squares of sides of length $1 / N$ and then into triangles by inserting the SW-NE diagonals. We imposed homogeneous Neumann data on the sides $x=0$ and $y=0$ and Dirichlet data on the sides $x=1$ and $y=1$. Therefore, the problem can be viewed as a Dirichlet problem over $[-1,1] \times[-1,1]$, and so our error analysis applies directly.

We computed our approximation (5.3) by solving the equivalent minimization problem (5.4). We used a Polak-Ribière conjugate gradient method, which worked reasonably well for the values of $p$ reported here. We did not experiment with the augmented Lagrangian approach advocated by Glowinski and Marrocco [5], but this conjugate gradient approach was far superior to the gradient method suggested by Wei [10].

For our test problems we consider solutions of the radially symmetric problem, Example 3.1, extended to the unit square. In the first three examples we took for various values of $p$ and $\gamma$

$$
\begin{aligned}
& f \equiv F(r) \equiv r^{\sigma} \quad \text { and } \\
& u \equiv U(r) \equiv(p-1)[1 /(\sigma+2)]^{1 /(p-1)}\left[1-r^{(\sigma+p) /(p-1)}\right] /(\sigma+p) .
\end{aligned}
$$

In all the examples, $f$ is sufficiently smooth, so that the error bounds for $u^{h}$ in the previous sections hold for $\hat{u}^{h}$ as well.

Example 5.1. This is (5.9) with $\sigma=0$ and $p=1.5$. It follows from (3.12) that $u \in W^{3,1}(\Omega) \cap C^{2,1}(\bar{\Omega})$, and so from Theorem 3.1 we expect $O(h)$ convergence in $W^{1,1.5}(\Omega)$. This is certainly achieved by inspecting Table 5.1 , where we adopt the notation $0.8233(-3) \equiv 0.8233 \times 10^{-3}$. In fact, $\hat{u}^{h}$ is converging to $u$ at the rate $O\left(h^{2}\right)$ in $L^{\infty}(\Omega)$, and there is a superconvergence for $\pi_{h} u-\hat{u}^{h}$ in $W^{1,1}(\Omega)$ and $W^{1, p}(\Omega)$.

TABLE 5.1

\begin{tabular}{|c|c|c|c|}
\hline$N$ & $\left\|\pi_{h} u-\hat{u}^{h}\right\|_{W^{1,1}(\Omega)}$ & $\left\|\pi_{h} u-\hat{u}^{h}\right\|_{W^{1, p}(\Omega)}$ & $\left\|\pi_{h} u-\hat{u}^{h}\right\|_{L^{\infty}(\Omega)}$ \\
\hline 10 & $0.8233(-3)$ & $0.4823(-3)$ & $0.8150(-3)$ \\
20 & $0.2061(-3)$ & $0.1207(-3)$ & $0.2034(-3)$ \\
40 & $0.5196(-4)$ & $0.3043(-4)$ & $0.5109(-4)$ \\
80 & $0.1235(-4)$ & $0.723(-5)$ & $0.1263(-4)$ \\
\hline
\end{tabular}


Example 5.2. This is (5.9) with $\sigma=0$ and $p=4$. It follows from Example 3.1 and $\S 4$ that $u \in W^{2, s}(\Omega)$, with $s<3$, and from (4.14c) we expect $O(h)$ convergence in $W^{1,1}(\Omega)$. From Table 5.2 we see this is achieved. In fact, $\pi_{h} u-\hat{u}^{h}$ exhibits superconvergence in $W^{1,1}(\Omega)$.

TABLE 5.2

\begin{tabular}{|c|c|c|c|}
\hline$N$ & $\left\|\pi_{h} u-\hat{u}^{h}\right\|_{W^{1,1}(\Omega)}$ & $\left\|\pi_{h} u-\hat{u}^{h}\right\|_{W^{1, p}(\Omega)}$ & $\left\|\pi_{h} u-\hat{u}^{h}\right\|_{L^{\infty}(\Omega)}$ \\
\hline 10 & $0.1789(-2)$ & $0.4486(-2)$ & $0.3790(-2)$ \\
20 & $0.5049(-3)$ & $0.2519(-2)$ & $0.1585(-2)$ \\
40 & $0.1376(-3)$ & $0.1414(-2)$ & $0.6493(-3)$ \\
80 & $0.3659(-4)$ & $0.7936(-3)$ & $0.2625(-3)$ \\
\hline
\end{tabular}

Example 5.3. Here we take (5.9) with $\sigma=7$ and $p=4$. It follows from Example 3.1 that $u \in W^{2, \infty}(\Omega)$. From (4.12), as $s=\infty$ and $\gamma<2 / 7$, we have with $q=1$ that $t>32 / 11$. Therefore, for all $\varepsilon>0$ we have that

$$
\left\|u-\hat{u}^{h}\right\|_{W^{1,1}(\Omega)} \leq C h^{(11-\varepsilon) / 16} .
$$

We note that a sharper bound, $h^{(14-\varepsilon) / 19}$, can be obtained by noting that for this model problem $\left|U^{\prime}(r)\right| \geq C r^{8 / 3}$ and applying (4.6) and (4.7) directly. From Table 5.3 we see that the above bounds are pessimistic. In fact, we have $O\left(h^{2}\right)$ convergence in $L^{\infty}(\Omega)$ and $\pi_{h} u-\hat{u}^{h}$ exhibits superconvergence in $W^{1,1}(\Omega)$.

TABLE 5.3

\begin{tabular}{|c|c|c|c|}
\hline$N$ & $\left\|\pi_{h} u-\hat{u}^{h}\right\|_{W^{1,1}(\Omega)}$ & $\left\|\pi_{h} u-\hat{u}^{h}\right\|_{W^{1, p}(\Omega)}$ & $\left\|\pi_{h} u-\hat{u}^{h}\right\|_{L^{\infty}(\Omega)}$ \\
\hline 10 & $0.8153(-3)$ & $0.5988(-2)$ & $0.5014(-2)$ \\
20 & $0.2164(-2)$ & $0.1931(-2)$ & $0.1235(-2)$ \\
40 & $0.5918(-3)$ & $0.8893(-3)$ & $0.2989(-3)$ \\
80 & $0.1429(-3)$ & $0.1952(-3)$ & $0.6449(-4)$ \\
\hline
\end{tabular}

Finally we consider an example for $p>2$, where $\{(x, y) \in \Omega: f(x, y)=0\}$ does not have zero measure.

Example 5.4. We take

$$
F(r) \equiv \begin{cases}0 & \text { for } r \leq a, \\ 4^{p-1}(r-a)^{(3 p-4)}[2+(a / r)-3 p] & \text { for } r \geq a\end{cases}
$$

and

$$
U(r) \equiv \begin{cases}0 & \text { for } r \leq a \\ (r-a)^{4} & \text { for } r \geq a\end{cases}
$$


with $a=0.3$ and $p=4$. At present the only global error estimate we have for this case is the result $(4.5 \mathrm{~b})$. Clearly, this is pessimistic from inspecting Table 5.4 , where once again we see $O\left(h^{2}\right)$ convergence in $L^{\infty}(\Omega)$ and $\pi_{h} u-\hat{u}^{h}$ is superconvergent in $W^{1,1}(\Omega)$. We note that the maximum error did not occur in the disc $\{r: r \leq 0.3\}$.

TABLE 5.4

\begin{tabular}{|c|c|c|c|}
\hline$N$ & $\left\|\pi_{h} u-\hat{u}^{h}\right\|_{W^{1,1}(\Omega)}$ & $\left\|\pi_{h} u-\hat{u}^{h}\right\|_{W^{1, p}(\Omega)}$ & $\left\|\pi_{h} u-\hat{u}^{h}\right\|_{L^{\infty}(\Omega)}$ \\
\hline 10 & $0.5879(-1)$ & $0.4653(-1)$ & $0.3080(-1)$ \\
20 & $0.1553(-1)$ & $0.1182(-1)$ & $0.7930(-2)$ \\
40 & $0.4332(-2)$ & $0.3118(-2)$ & $0.1992(-2)$ \\
80 & $0.1139(-2)$ & $0.1706(-2)$ & $0.4923(-3)$ \\
\hline
\end{tabular}

\section{BIBLIOGRAPHY}

1. C. Atkinson and C. R. Champion, Some boundary-value problems for the equation $\nabla \cdot\left(|\nabla \phi|^{N} \nabla \phi\right)=0$, Quart. J. Mech. Appl. Math. 37 (1984), 401-419.

2. C. Atkinson and C. W. Jones, Similarity solutions in some non-linear diffusion problems and in boundary-layer flow of a pseudo plastic fluid, Quart. J. Mech. Appl. Math. 27 (1974), 193-211.

3. S.-S. Chow, Finite element error estimates for non-linear elliptic equations of monotone type, Numer. Math. 54 (1989), 373-393.

4. P. G. Ciarlet, The finite element method for elliptic problems, North-Holland, Amsterdam, 1978.

5. R. Glowinski and A. Marrocco, Sur l'approximation par éléments finis d'ordre un, et la résolution, par pénalisation-dualité, d'une classe de problèmes de Dirichlet non lineaires, RAIRO Anal. Numér. 2 (1975), 41-76.

6. A. Kufner, O. John, and S. Fučik, Function spaces, Noordhoff, Leyden, 1977.

7. G. M. Lieberman, Boundary regularity for solutions of degenerate elliptic equations, Nonlinear Anal. 12 (1988), 1203-1219.

8. J. R. Philip, N-diffusion, Austral. J. Phys. 14 (1961), 1-13.

9. V. B. Tyukhtin, The rate of convergence of approximation methods of solution of one-sided variational problems. I, Vestnik Leningrad. Univ. Mat. Mekh. Astronom. 13 (1982), 111113.

10. D. Wei, Finite element approximations of solutions to p-harmonic equations with Dirichlet data, Numer. Funct. Anal. Optim. 10 (1989), 1235-1251.

Department of Mathematics, Imperial College, London SW7 2BZ, England

E-mail address: j.barrett@ic.ac.uk 